\title{
The Evolution of Compact Steep-Spectrum Sources
}

\author{
S. Jeyakumar \& D. J. Saikia
}

National Centre for Radio Astrophysics, Tata Institute of Fundamental Research, Pune 411007, India

\begin{abstract}
We used our high-resolution radio images made with VLA of a large number of compact steep-spectrum (CSS) sources selected from well-defined samples of radio sources and the radio data of these samples to explore the evolution of CSSs with time. We suggest that the majority of CSSs are likely to be young sources advancing outwards through an asymmetric inhomogeneous environment to form the larger ones. We examine the symmetry parameters of both CSSs and larger sources and compare these with both analytical and numerical estimates of propagation of jets through an asymmetric environment. We also examine the consistency of CSSs with the unified scheme for radio galaxies and quasars.
\end{abstract}

Introduction: We have estimated the symmetry parameters of CSS objects to understand the evolution of these objects and the consistency of their properties with the orientation based unification scheme. We define the CSSs to be with a size $<20 \mathrm{kpc}\left(H_{0}=100 \mathrm{~km} \mathrm{~s}^{-1} \mathrm{Mpc}^{-1}, q_{0}=0\right)$ and with steep high frequency radio spectra $\alpha>0.5\left(S \propto \nu^{-\alpha}\right)$.

We have chosen sources with luminosity $P_{178 M H z}>10^{26} \mathrm{~W} \mathrm{~Hz}^{-1} \mathrm{sr}^{-1}$ from the complete 3CR (Laing, Ryle, \& Longair 1983) sample and 3C and Peacock \& Wall (1982) sample and the complete sample of S4 (Kapahi 1981, Stickel \& Kühr 1994) radio sources with luminosity $P_{5 G H z} \geq 5 \cdot 10^{24} \mathrm{~W} \mathrm{~Hz}^{-1} \mathrm{sr}^{-1}$ and $\alpha_{408}^{5000} \geq 0.5$. We have used only the sources with detected core to estimate the symmetry parameters reliably. The deficiency of symmetric quasars and the higher fraction of core emission of quasars in our sample indicate the consistency of CSSs with the unification scheme (Saikia et al. 1995, Saikia et al. 1996). However, the brighter lobe nearer to the core, sources below $\log \left(r_{L}\right)=0$ line in fig-2(a), indicate the role of an asymmetric environment in most of the sources.

Jet propagation through asymmetric environments: We assume the jets on both sides have equal power and evolve into different ISMs. We calculate the distance traveled by the head of the jet, moving subrelativistically, at various times, using the pressure balance between the jet momentum flux density and the ram pressure of the external medium (Scheuer 1974, GopalKrishna \& Wiita 1991) $n(D)(d D / d t)^{2}=4 K L_{b} /\left(\pi \mu m_{h}(D \theta)^{2}\right)$ where K - efficiency factor, $L_{b}$ - beam power and $\theta$ - opening angle and the luminosity ratio $r_{L}=\left(n\left(D_{1}\right) / n\left(D_{2}\right)\right)^{m}, m=3(1+\alpha) / 10$ (Eilek \& Shore 1989).

We consider the more realistic ambient density profile, falling off till a distance $R_{3}=R_{4}$ in the ISM for both the jets and a constant ICM density beyond $R_{3}$ and $R_{4}$. The asymmetry is introduced in the core region till a distance of $R_{1}$ and $R_{2}\left(R_{1}=R_{2}\right)$ by different power law with $\delta_{1}=0.675, \delta_{2}=0.3$ and $\delta=0.75$ fig $1(\mathrm{a})$. Till the lobes are within the asymmetric medium the luminosity ratio, and the distance ratio become more asymmetric fig-1(b). They eventually approach unity, when the jets move away into symmetric medium fig-1(c).

We simulated the propagation of jets through asymmetric environments using the ZEUS-2D code (Stone \& Norman 1992; Hooda, Mangalam, \& Wiita 1994). We simulated for two jets traveling in an asymmetric medium explained above with $R_{1}=R_{2}=5 \mathrm{kpc}, R_{3}=R_{4}=25 \mathrm{kpc}$. The distance ratio $r_{D}$ is given in fig-2(b), for a jet with mach number of 26.0 and initial jet density of 0.001 of 

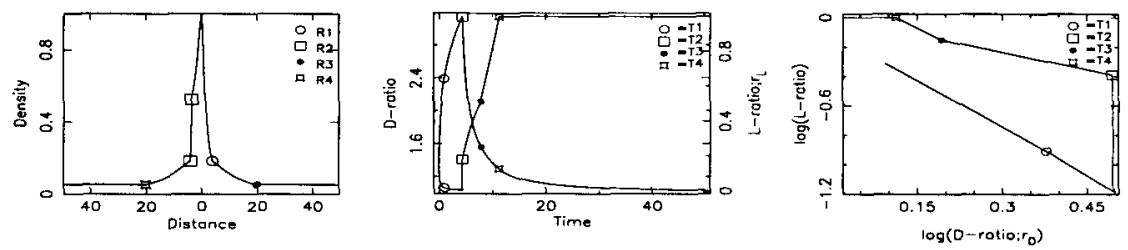

Figure 1. Analytical model: density (a), $r_{D}$ and $r_{L}$ (b), $r_{D}-r_{L}$ (c).
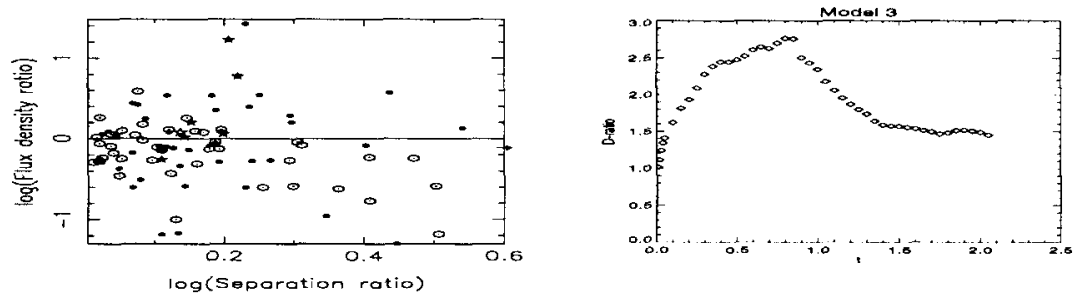

Figure 2. (a) observed $r_{D}$ (Separation ratio) $-r_{L}$ (Flux density ratio) diagram filled - quasars, unfilled - galaxies; (b) simulated $r_{D}$ with time.

the ambient density. The symmetrization is seen in these simulations when the jets cross the asymmetric medium.

Conclusion: Our analytical calculations and numerical simulations show that many of the CSSs are evolving in an asymmetric environment even though they are consistent with the orientation based unification scheme. Comparison of larger sources indicates that most of the larger sources could have passed through such a phase. These results imply that most of the CSS sources are young.

Acknowledgments. S. Jeyakumar thanks the organizing committee of IAU 164, CSIR of India and NCRA for the financial support for participating in IAU 164.

\section{References}

Eilek, J. A., \& Shore, S. N. 1989. ApJ, 342, 187-207.

Gopal-Krishna \& Wiita, P. J. 1991. ApJ, 373, 325-335.

Hooda, J. S., Mangalam, A. V., \& Wiita, P. J. 1994. ApJ, 423, 116-130.

Kapahi, V. K. 1981. A\&AS, 43, 381-393.

Laing, R. A., Riley, J. M., \& Longair, M. S. 1983. MNRAS, 204, 151-187.

Peacock, J. A., \& Wall, J. V. 1982. MNRAS, 198, 843-860.

Saikia, D. J., et al.1995. MNRAS, 276, 1215-1223.

Saikia, D. J., et al.1996. In Second Workshop on Gigahertz Peaked Spectrum and Compact Steep Spectrum Radio Sources, eds. I. A. G. Snellen, R. T. Schilizzi, H. J. A Röttgering, \& M. N. Bremer (Leiden: Leiden Obs.), 252-262.

Scheuer, P. A. G. 1974. MNRAS, 166, 513-528.

Stickel, M., \& Kühr, H. 1994. A\&AS, 103, 349-363.

Stone, J. M., \& Norman, M. L. 1992. ApJS, 80, 753-790. 